\title{
INTEGRAÇÃO NO ENSINO DE DISCIPLINAS MATEMÁTICAS E DE PROGRAMAÇÃO: UMA REVISÃO SISTEMÁTICA
}

INTEGRATION IN THE TEACHING OF MATHEMATICAL AND PROGRAMMING SUBJECTS: A SYSTEMATIC REVIEW Karoline Guedes ${ }^{1}$, Antonio Henrique Meira ${ }^{2}$, Carlos Roberto Beleti Junior ${ }^{3}$, Juliana Verga Shirabayashi ${ }^{4}$

DOI: 10.37702/REE2236-0158.v39p379-389.2020

\section{RESUMO}

A integração curricular das disciplinas em cursos de Engenharia pode ser fundamental para o processo de aprendizado, pois, compreendendo a importância de tais disciplinas, o estudante tende a ter um maior comprometimento e engajamento. Assim, a integração de disciplinas - como Algoritmos e Programação de Computadores às disciplinas matemáticas da grade curricular do núcleo base de cursos de graduação de Engenharia - pode ser uma forma de melhorar o ensino e aprendizagem, consequentemente otimizando o desempenho dos estudantes em tais disciplinas. Dessa forma, o objetivo deste trabalho é apresentar uma revisão sistemática sobre a interdisciplinaridade em disciplinas nos cursos de Engenharia a partir de periódicos e bases de dados nacionais. Embora este assunto seja de grande relevância, existem ínfimos trabalhos e pesquisas que destacam a interdisciplinaridade ou integração curricular existente em cursos de graduação, principalmente em cursos de Engenharia.

Palavras-chave: interdisciplinaridade; integração curricular; Programação e Matemática.

\begin{abstract}
The curricular integration of disciplines in engineering courses can be fundamental to the learning process, since understanding the importance of these disciplines, the student tends to have a greater commitment and engagement. Thus, the integration of disciplines such as algorithms and computer programming to the mathematical disciplines of the core curriculum of the base of undergraduate engineering courses, can be a way to improve teaching and learning, consequently Optimizing the performance of students in such disciplines. Thus, the objective of this work is to present a systematic review about interdisciplinarity in disciplines in engineering courses from national journals and databases. Although this issue is of great relevance, there are few studies and researches that highlight the interdisciplinarity or curricular integration existing in undergraduate courses, especially in engineering courses.
\end{abstract}

Keywords: interdisciplinarity; curricular integration; Programming and Mathematics.

\footnotetext{
${ }^{1}$ Mestranda em Engenharia de Produção, Universidade Estadual de Maringá; karolineguedes13@ gmail.com

${ }^{2}$ Graduado em Engenharia de Produção, Universidade Federal do Paraná campus Avançado em Jandaia do Sul; antoniorick1997@gmail.com

${ }^{3}$ Doutorando em Educação, Professor da Universidade Federal do Paraná campus Avançado em Jandaia do Sul; carlosbeleti@ufpr.br

${ }^{4}$ Doutora em Engenharia Elétrica, Professora da Universidade Federal do Paraná campus Avançado em Jandaia do Sul; juliana.verga@ufpr.br
} 


\section{INTRODUÇÃO}

A formação de profissionais com alta capacidade produtiva é essencial para a economia e desenvolvimento de um país. A qualidade com que a universidade prepara seus estudantes é uma questão que deve ser estudada e debatida, pois estes serão os futuros profissionais que atuarão nas empresas e organizações e que terão influência na situação social e econômica de uma nação.

A adesão do saber tecnológico em qualquer profissão possibilita maior adaptação às constantes mudanças e permite melhor desenvoltura em uma sociedade cada vez mais digital. Assim, em diversas áreas, grandes resultados podem ser obtidos a partir da integração do saber técnico com o saber tecnológico, facilitando o aprendizado na graduação, a fixação do conteúdo e a implementação da teoria na prática.

$\mathrm{O}$ ensino de programação em cursos de Engenharia é obrigatório na maioria das grades curriculares, segundo as Diretrizes Curriculares Nacionais dos Cursos de Graduação de Engenharia (BRASIL, 2002). Porém, por vezes, o que é ministrado isola as disciplinas, criando "ilhas" de conhecimento e limitações no uso dos conceitos abordados. A integração de disciplinas de algoritmos com outras disciplinas do currículo de cursos de Engenharia pode auxiliar o ensino e aproximar as zonas de conhecimento do estudante, facilitando assim o aprendizado.

Conforme observado por meio do desempenho dos estudantes da Universidade Federal do Paraná, Campus Jandaia do Sul, em disciplinas de Algoritmos e Programação de Computadores, relacionando-as com disciplinas subsequentes, em especial disciplinas matemáticas como Cálculo e Pesquisa Operacional, nota-se que estudantes que apresentam desempenho insatisfatório naquelas disciplinas possuem desempenho indesejável também nestas.

Com o exposto, este trabalho tem o objetivo de apresentar uma revisão sistemática sobre a integração de disciplinas de Algoritmos e Programação de Computadores com disciplinas correlatas que possuem características matemáticas e tecnológicas, tais como Cálculo Diferencial e Integral, Cálculo Numérico e Pesquisa Operacional.

\section{METODOLOGIA}

A revisão sistemática da literatura é utilizada para avaliar e interpretar toda a pesquisa disponível e relevante para uma questão de pesquisa específica, área de tópico ou fenômeno de interesse. Para isso, é necessária a utilização de uma metodologia confiável, rigorosa e auditável. Para a realização deste trabalho foram consideradas algumas etapas, visando a estruturar uma Revisão Sistemática, a qual se baseou nas etapas dispostas por Kitchenham (2004), apresentadas na Figura 1.

Figura 1 - Fases da Revisão Sistemática

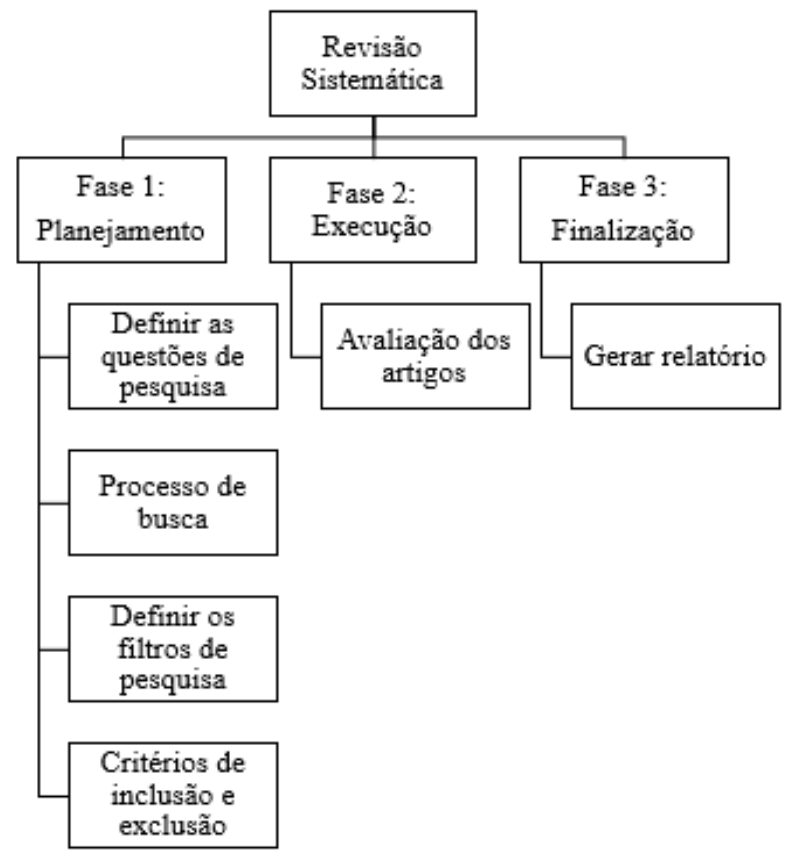

Fonte: adaptada de Kitchenham (2004).

\section{REVISÃO SISTEMÁTICA}

Com o passo a passo da metodologia definido neste trabalho, serão apresentadas nas próximas seções uma breve descrição do significado e da importância de cada etapa, seguida pela condução da revisão realizada.

a) Questões de pesquisa: são delineadoras quanto ao objetivo de realização de uma revisão sistemática, pois definem a dimensão da 
pesquisa. As palavras-chave são fundamentais para a formação das strings de busca.

b) Processo de busca - PICOC (Population, Intervention, Comparation, Outcome, Context): é a etapa para conceber e gerenciar as informações utilizadas nas buscas por trabalhos na área de pesquisa foco. É essencial organizar e manter os dados definidos nessa etapa, pois são norteadores durante as buscas nas bases de dados.

c) Strings e bases de busca: a combinação entre palavras-chave forma as chamadas strings de busca, utilizadas nas pesquisas nas bases de dados selecionadas. As bases são periódicos nacionais de relevância na área de Ensino de Ciência, Tecnologia e Engenharias. O motor de busca foi selecionado por ser uma ferramenta de busca de informações de caráter acadêmico e por apresentar informações relativas ao impacto dos trabalhos por meio do número de citações recebidas, além de catalogar produções de veiculação gratuita.

d) Filtros de pesquisa, critério de seleção e estratégia de busca: com base na etapa que compreende o PICOC, foram determinados critérios de seleção para inclusão dos trabalhos a serem considerados nesta revisão. Para complementar tais critérios, uma estratégia de busca compreendendo três fases também foi utilizada para categorizar e organizar os trabalhos objetos de estudo na revisão.

e) Resultados obtidos: como etapa final, os trabalhos selecionados foram sistematizados com objetivo de extrair informações de relevância para a área de estudo desta revisão.

\section{Fase 1 - Planejamento}

Para iniciar as pesquisas desta revisão, foram elaboradas questões centrais a serem abordadas. Para cada questão, palavras-chave foram definidas para a formação das strings de busca. Tanto as questões abordadas quanto as palavras-chave são fundamentais para a realização da revisão sistemática. A correta determinação das palavras-chave tende a garantir um bom retorno nos mecanismos de buscas. Dentre diversos questionamentos elencados, dois foram considerados, conforme a seguir: a) A influência (ou correlação) do estudo de Algoritmos e Programação de Computadores no desempenho de disciplinas da área de Exatas em cursos de Engenharia.

b) A relevância do aprendizado de Algoritmos e Programação em disciplinas subsequentes, tais como Cálculo Diferencial e Integral, Cálculo Numérico e Pesquisa Operacional.

A partir das indagações, formaram-se palavras-chave de relevância para o objetivo das buscas nos periódicos e bases de dados. Abaixo encontram-se as palavras-chave utilizadas, agrupadas respectivamente para cada questionamento realizado:

a) Influência; Algoritmos; Programação de Computadores; Desempenho acadêmico; Engenharias; Correlação; Interdisciplinaridade.

b) Interdisciplinaridade; Programação de Computadores; Engenharia; Pesquisa Operacional; Tecnologia da Decisão; Aprendizado; Correlação; Influência; Cálculo Numérico.

Após a definição das questões de pesquisa e palavras-chave, foi determinado o processo de busca. O termo PICOC foi um formato simples de definição de sistemas de pesquisas e/ou intervenções. Ele consiste em cinco tópicos básicos para a pesquisa: Population (população de interesse); Intervention (características de interesse); Comparation (comparação básica das características de interesse); Outcome (objetivo, razão pela qual é realizado o estudo); Context (circunstâncias nas quais está sendo realizado o estudo).

É de extrema importância que os objetivos, restrições e limitações de qualquer estudo sejam bem definidos a fim de maximizar a probabilidade de sucesso. Dessa forma, definiram-se os cinco tópicos para esta pesquisa, conforme segue:

1) Population: cursos de graduação no Brasil, especialmente Engenharias.

2) Intervention: desempenho nas disciplinas de Algoritmos e Programação de Computadores e disciplinas correlatas.

3) Comparation: desempenho nas disciplinas de Cálculo Diferencial e Integral, Cálculo Numérico e Pesquisa Operacional com disciplinas de 
Algoritmos e Programação de Computadores e correlatas.

4) Outcome: gerar soluções para a melhoria do aprendizado de Algoritmos a partir da interdisciplinaridade entre disciplinas de cunho formativo base.

5) Context: cursos brasileiros de Engenharia (principalmente Engenharia de Produção) cadastrados no currículo base do MEC.

A definição da composição das strings de busca por meio das palavras-chave bem como as bases de buscas a serem averiguadas são fundamentais para delimitar a abrangência e profundidade do estudo. Idealiza-se um arranjo entre as palavras-chave com sentido e significância entre o determinado em PICOC assim como a escolha das bases de busca. As seguintes strings de busca foram então utilizadas:

a) (Influência ou correlação) and (algoritmos ou programação de computadores) and (desempenho acadêmico ou interdisciplinaridade) and (cursos de engenharia ou disciplinas de cálculo).

b) (Influência ou vantagem ou correlação) and (aprendizado ou estudo ou experiência) and (interdisciplinaridade) and (algoritmo ou programação de computadores) and (cálculo numérico ou pesquisa operacional) and (engenharia).

As bases de dados neste estudo foram apontadas utilizando-se alguns critérios como: nacionalidade do estudo (preferencialmente brasileiro), disponibilidade on-line e acesso livre. Definiu-se a utilização das seguintes bases de dados:

a) Revista de Ensino de Engenharia ${ }^{5}$ e Anais do Congresso Brasileiro de Ensino em Engenharia (COBENGE) ${ }^{6}$.

b) Revista de Ensino de Ciência e Engenharia ${ }^{7}$.

c) Revista Brasileira de Ensino de Ciência e Tecnologia ${ }^{8}$.

d) Revista Brasileira de Informática na Educação ${ }^{9}$.

e) Revista Tecnologias na Educação (Tecedu $)^{10}$.

\footnotetext{
${ }^{5}$ http://revista.educacao.ws/revista/index.php/abenge/index.

${ }^{6}$ http://www.abenge.org.br/publicacoes.php

${ }^{7} \mathrm{http}$ ://www.latec.ufrj.br/revistas/index.php?journal=ensinodeciencia

${ }^{8}$ https://revistas.utfpr.edu.br/rbect.
}

f) Revista Novas Tecnologias na Educação (Renote) ${ }^{11}$.

g) Google acadêmico ${ }^{12}$.

Depois de realizadas as buscas, foram definidos os critérios para seleção dos artigos a serem considerados na revisão sistemática. São eles:

1) trabalhos disponíveis na internet e de forma gratuita;

2) artigos publicados em revistas, congressos, dissertações ou teses;

3) artigos escritos em português;

4) preferência por estudos realizados no Brasil.

\section{Fase 2 - Execução}

Com tais critérios, definiu-se ainda a estrutura para categorizar a seleção e avaliação dos artigos dos trabalhos buscados, conforme apresenta-se na Figura 2.

Primeiramente (Etapa 1) foram utilizados as strings e os trabalhos com palavras-chave condizentes com os objetivos deste trabalho. Foram selecionados conforme o título, o resumo e o tipo de documento (artigo, dissertação, etc.) (Etapa 2). Eliminaram-se as pesquisas que se diferem quanto ao foco do trabalho ou que não satisfizeram os critérios de seleção. Os trabalhos foram então analisados baseando-se na "Introdução" e "Conclusão" (Etapa 3), o que permitiu a eliminação daqueles em que a metodologia ou objetivo não corresponderam ao tema requerido. Por fim (Etapa 4), realizou-se a leitura na íntegra dos trabalhos, avaliando se estavam condizentes com o objetivo do estudo; em caso afirmativo, foram aceitos.

\footnotetext{
${ }^{9}$ http://www.br-ie.org/pub/index.php/rbie.

${ }^{10} \mathrm{http}: / /$ tecedu.pro.br/.

${ }^{11} \mathrm{http}: / /$ seer.ufrgs.br/renote.

${ }^{12}$ https://scholar.google.com.br/.
} 
Figura 2 - Etapas da seleção e avaliação dos artigos.

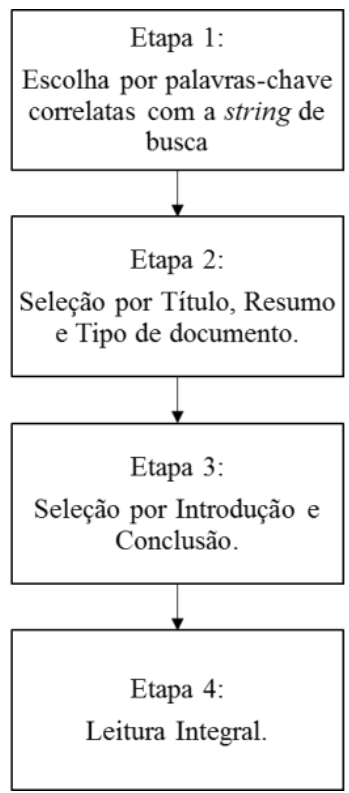

Fonte: elaborada pelos autores.

A Tabela 1 apresenta a relação total de trabalhos encontrados e selecionados em cada etapa de busca. As demais bases de busca não retornaram resultados satisfatórios ou não apresentaram trabalhos que tenham passado pela Etapa 3.

Tabela 1 - Número de trabalhos retornados em cada etapa

\begin{tabular}{ccccc}
\hline $\begin{array}{c}\text { Base de } \\
\text { Busca }\end{array}$ & $\begin{array}{c}\text { Trabalhos } \\
\text { Encontrados }\end{array}$ & $\begin{array}{c}\text { Etapa } \\
\mathbf{1}\end{array}$ & $\begin{array}{c}\text { Etapa } \\
\mathbf{2}\end{array}$ & $\begin{array}{c}\text { Etapa } \\
\mathbf{3}\end{array}$ \\
\hline COBENGE & 10 & 4 & 4 & 3 \\
\hline Renote & 12 & 4 & 1 & 1 \\
\hline Tecedu & 15 & 1 & 1 & 1 \\
\hline $\begin{array}{c}\text { Google } \\
\text { Acadêmico }\end{array}$ & 423 & 49 & 19 & 13 \\
\hline Fon
\end{tabular}

Fonte: Elaborado pelos autores.

\section{Fase 3 - Finalização}

A Tabela 2 exibe os trabalhos após a Etapa 3 e apresenta uma síntese das informações de cada pesquisa.

Tabela 2 - Trabalhos selecionados e sínteses

PRETO et al., 2005 O trabalho apresenta a análise de uma abordagem voltada para aplicações matemáticas, em comparação a uma abordagem geral envolvendo programação.

FILHO et al., 2012 O trabalho mostra a correlação do uso da tecnologia e desempenho acadêmico de disciplinas em Engenharia.

COUTINHO; LIMA; O trabalho analisa a necessidade de um melhor aprendizado lógico para o bom desempenho SANTOS, 2017 do estudante em disciplinas de programação.

MOTA, 2011 O trabalho aborda a integração de softwares com a disciplina de Cálculo Numérico.

CORDENONZI et al., O trabalho mostra a interdisciplinaridade entre Cálculo, Algoritmo e Lógica, promovendo 2015 maior dinâmica na aula e engajamento dos estudantes.

aprendizado de Cálculo Numérico em um curso de Engenharia. Destaca também a importância do aprendizado de programação para o sucesso na utilização de softwares relacionados.

SOUSA, 2014

O trabalho avalia as contribuições que o software LOpt oferece quando utilizado como instrumento de apoio na resolução de problemas de Programação Linear.

AMARAL, LEITE, O trabalho propõe a construção de códigos didáticos em Scilab para implementação de SILVA, 2013 técnicas numéricas a fim de modernizar a disciplina de Cálculo Numérico e motivar a aprendizagem dos estudantes.

PAZETO, PRIETCH, O trabalho mostra as experiências e propostas de projetos interdisciplinares no Projeto 2010 Pedagógico do Curso de Licenciatura em Computação. 
PRETO, SCHEER, O trabalho apresenta sugestões para melhorar o ensino de Matemática em cursos de 2004 Engenharia, por meio de softwares e maior integração com outras disciplinas do curso.

SILVA, SOUZA, O trabalho mostra a importância da utilização da programação computacional no ensino de SILVA, $2016 \quad$ Matemática.

CONCILIO, O trabalho mostra a integração de programação com outras disciplinas, gerando interesse SOARES, $2001 \quad$ no estudante.

GUIMARÃES, O trabalho exibe um estudo de caso que aponta os benefícios do uso de softwares DUARTE, 2007 matemáticos para o aprendizado de Pesquisa Operacional.

AMBRÓSIO et al., $\quad$ O trabalho apresenta um estudo sobre as diferenças nos processos de aprendizado dos 2011 estudantes considerados "bons" e "ruins".

ZAINA, CAVERSAN, 2005

O trabalho aponta questões motivacionais na integração de disciplinas correlatas.

\begin{tabular}{cl} 
MÜLLER, 2000 & $\begin{array}{c}\text { O trabalho mostra as tendências interdisciplinares necessárias para o aprendizado de } \\
\text { disciplinas de Matemática. }\end{array}$ \\
\hline $\begin{array}{c}\text { GOMES, } \\
\text { HENRIQUES, }\end{array}$ & $\begin{array}{c}\text { O trabalho exibe um panorama geral da importância do aprendizado de programação e } \\
\text { formas para reduzir as dificuldades de aprendizado dos estudantes. }\end{array}$ \\
\hline CASTRO et al., 2002 2008 & $\begin{array}{c}\text { O trabalho discute o desafio de ensinar programação nos cursos introdutórios de graduação } \\
\text { e diferentes abordagens utilizadas com tal finalidade. }\end{array}$ \\
\hline
\end{tabular}

Fonte: elaborada pelos autores.

A Tabela 3 foi produzida para apresentar a relação dos trabalhos organizados por temas relacionados em cada artigo selecionado.

Tabela 3 - Divisão por temas relacionados

\begin{tabular}{|c|c|c|c|c|c|c|}
\hline Referência & $\begin{array}{l}\text { Interdiscipli- } \\
\text { naridade }\end{array}$ & Didática & $\begin{array}{l}\text { Programação/ } \\
\text { ferramentas }\end{array}$ & Cálculo & $\begin{array}{l}\text { Cálculo } \\
\text { numérico }\end{array}$ & $\begin{array}{c}\text { Pesquisa } \\
\text { operacional }\end{array}$ \\
\hline \multicolumn{7}{|l|}{ PRETO et al., 2005} \\
\hline \multicolumn{7}{|l|}{ FILHO et al., 2012} \\
\hline \multicolumn{7}{|l|}{$\begin{array}{l}\text { COUTINHO, LIMA, } \\
\text { SANTOS, } 2017\end{array}$} \\
\hline \multicolumn{7}{|l|}{ MOTA, 2011} \\
\hline \multicolumn{7}{|l|}{$\begin{array}{l}\text { CORDENONZI et al., } \\
2015\end{array}$} \\
\hline \multicolumn{7}{|l|}{ SILVA, 2013} \\
\hline \multicolumn{7}{|l|}{ SOUSA, 2014} \\
\hline $\begin{array}{l}\text { AMARAL, LEITE, } \\
\text { SILVA, } 2013\end{array}$ & & & & & & \\
\hline
\end{tabular}




\begin{tabular}{|c|c|c|c|c|}
\hline $\begin{array}{c}\text { PAZETO, PRIETCH, } \\
2010\end{array}$ & $\checkmark$ & $\checkmark$ & $\checkmark$ & \\
\hline PRETO, SCHEER, 2004 & $\checkmark$ & $\checkmark$ & $\checkmark$ & $\checkmark$ \\
\hline $\begin{array}{l}\text { SILVA, SOUZA, SILVA, } \\
2016\end{array}$ & $\checkmark$ & & $\checkmark$ & $\checkmark$ \\
\hline $\begin{array}{c}\text { CONCILIO, SOARES, } \\
2001\end{array}$ & $\checkmark$ & & $\checkmark$ & $\checkmark$ \\
\hline $\begin{array}{c}\text { GUIMARÃES, DUARTE, } \\
2007\end{array}$ & $\checkmark$ & & $\checkmark$ & \\
\hline AMBRÓSIO et al., 2011 & & $\checkmark$ & $\checkmark$ & \\
\hline $\begin{array}{l}\text { ZAINA, CAVERSAN, } \\
2005\end{array}$ & $\checkmark$ & $\checkmark$ & $\checkmark$ & \\
\hline MÜLLER, 2000 & & $\checkmark$ & & $\checkmark$ \\
\hline $\begin{array}{l}\text { GOMES, HENRIQUES, } \\
\text { MENDES, } 2008\end{array}$ & & $\checkmark$ & $\checkmark$ & \\
\hline CASTRO et al., 2002 & & $\checkmark$ & $\sqrt{ }$ & \\
\hline
\end{tabular}

Fonte: elaborada pelos autores.

Para uma melhor visualização quanto ao ano de publicação dos artigos, foi produzida a Figura 3, na qual se encontram os trabalhos selecionados divididos por ano de publicação, de 2000 a 2017.

Figura 3 - Número de trabalhos por ano

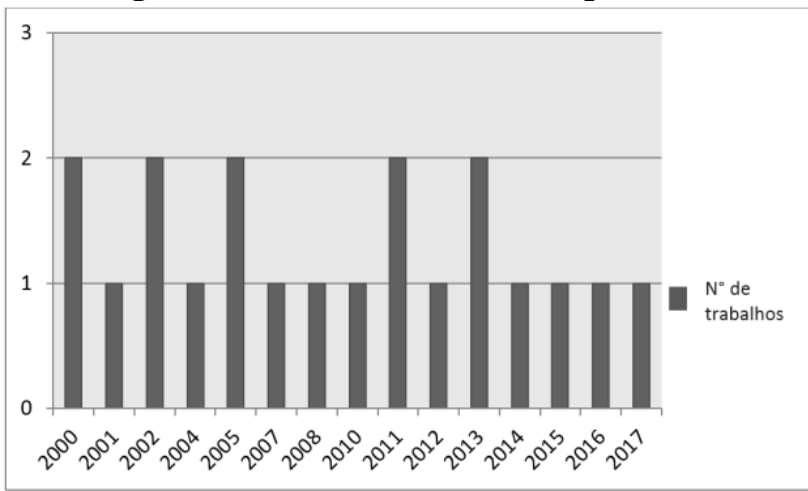

Fonte: elaborada pelos autores.

Importante observar a quantidade reduzida de trabalhos publicados em todo o intervalo de tempo, especialmente nos últimos quatro anos.

\section{RESULTADOS}

No estudo feito por Preto e Scheer (2004) em disciplinas do curso da Universidade Federal do Paraná, no curso de Engenharia da Computação, os resultados são animadores: melhor aproveitamento dos estudantes nas disciplinas envolvidas; prática das habilidades de trabalho em equipe; melhor compreensão da relação entre disciplinas e da importância de cada; aumento da compreensão do universo de problemas de Engenharia que podem ser resolvidos pela programação. Um estudo realizado pelo mesmo autor em conjunto com outros, em 2005 (PRETO et al., 2005), apresentou novamente bons resultados. Nesse estudo, foram analisadas as vantagens que duas linguagens de programação possuem no desafio de integração. A primeira linguagem, o Matlab, por não requerer maiores conhecimentos de programação, possibilita a concentração em aspectos como a conceituação do problema e 
suas aplicações. Na segunda linguagem de programação, a linguagem $\mathrm{C}$, é necessário maior foco em técnicas avançadas de programação, porém existe grande possibilidade da interação entre as disciplinas de outras áreas da Matemática (como Pesquisa Operacional), o que motiva o estudante, que compreenderá a relação entre as disciplinas, podendo resolver problemas diversos por meio da lógica de programação.

Os estudos de SILVA (2013), MOTA (2011) e AMARAL, LEITE, SILVA (2013) apresentam grandes resultados na utilização do software Scilab para o aprendizado de Cálculo Numérico. O estudo aponta, ainda, que a união da ferramenta tecnológica ajuda a didática em aula e engaja o estudante, facilitando o aprendizado e reduzindo o número de evasão e reprovação.

Outro estudo que apresenta resultados interessantes é o de ZAINA, CAVERSAN (2005) que apresenta um caráter motivador da interdisciplinaridade, em suas palavras:

tanto o caráter multidisciplinar como o interdisciplinar da experiência permitiu que o estudante não visualizasse a aprendizagem dos conceitos das duas disciplinas envolvidas de forma fragmentada. Permitiu o afloramento de processos internos de amadurecimento e de desenvolvimento dentro do contexto da aprendizagem da programação, que só podem ocorrer quando o estudante interage com aspectos múltiplos dentro de um eixo de aprendizagem (ZAINA; CAVERSAN, 2005, p. 8).

Um estudo bem relevante para a revisão foi também o de SOUSA (2014), no qual foi realizada uma análise da influência do uso de um software no desempenho acadêmico e engajamento com a disciplina de Pesquisa Operacional.

\section{CONSIDERAÇÕES FINAIS}

Com a revisão sistemática percebe-se, primeiramente, que a integração de disciplinas é aceita pelos autores, e também que a junção entre disciplinas de Algoritmos e Programação de Computadores com disciplinas correlatas apresenta resultados expressivos no desempenho acadêmico dos estudantes, como pode-se constatar em PRETO; SCHEER (2004); PRETO et al. (2005); CASTRO et al. (2002); MÜLLER (2000); SILVA, SOUZA, SILVA (2016); SILVA (2013); CORDENONZI et al. (2015).

Com esta revisão, fica evidente a importância da integração de disciplinas e os benefícios da interdisciplinaridade de programação, com disciplinas correlatas de Engenharia. Porém, não foram encontrados artigos relacionados exatamente com o tema proposto da revisão sistemática, levando em consideração as strings utilizadas, critérios de seleção e bases de buscas.

Um estudo mais profundo e detalhado sobre a importância e influência de programação em disciplinas de grande dependência de Lógica e Algoritmos, como Cálculo Numérico e Pesquisa Operacional, é altamente recomendado para trabalhos futuros.

\section{REFERÊNCIAS}

AMARAL, T. R.; LEITE, N. M. G.; SILVA, A. O. O ensino de Cálculo Numérico utilizando o Scilab. 2013. 9 p. Anais... VI Congresso internacional de ensino de matemática (Comunicação cientifica) - ULBRA, CanoasRio Grande do Sul, 2013. 4. Disponível em: <http://www.conferencias.ulbra.br/index.php/ ciem/vi/paper/view/1232/373>. Acesso em: 27 out. 2017.

AMBRÓSIO, A. P. L. et al. Programação de Computadores: compreender as dificuldades de aprendizagem dos alunos. Revista Galegoportuguesa de Psicoloxía e Educación, v. 19, n. 1, p. 185-197. [S.1.], 2011.

BRASIL. Ministério da Educação. Diretrizes Curriculares Nacionais para os Cursos de Engenharia. Parecer CES 1.362/2001, homologação publicada no DOU 25/02/2002, Seção 1, p. 17. Resolução CES/CNE 11/2002, publicada no DOU 09/04/2002, Seção 1, p. 32. 2002.

CASTRO, T. H. C. et al. Utilizando Programação Funcional em Disciplinas Introdutórias de Computação. 2002. 12 p. - (Departamento de Informática) - Universidade Federal do 
Espírito Santo, [S.1.], 2002. Disponível em: <http://www.lbd.dcc.ufmg.br/colecoes/wei/20 02/0015.pdf >. Acesso em: 27 out. 2017.

CONCILIO, R.; SOARES, M. V. Interdisciplinaridade um exemplo de aplicação bem sucedida. 2001. Anais... Congresso Brasileiro de Ensino em Engenharia. p. 239$245 . \quad$ Disponível em: <http://198.136.59.239/ abengeorg/Cobenge Anteriores/2001/trabalhos/MTE064.pdf >. Acesso em: 27 out. 2017.

CORDENONZI, W. et al. Uma Experiência Interdisciplinar no Ensino de Algoritmos e Matemática em um Contexto Binacional. 2015. Anais... Workshops do IV Congresso Brasileiro de Informática na Educação (CBIE 2015) - Instituto Federal Sul-rio-grandense, p. 1408-1417. [S.1.], 2015. Disponível em: $<$ http://www.br-

ie.org/pub/index.php/wcbie/article/view/6319/ 4428>. Acesso em: 27 out. 2017.

COUTINHO, E. F.; LIMA, E. T.; SANTOS, C. C. Um panorama sobre o desempenho de uma disciplina inicial de programação em um curso de graduação. Tecnologias na Educação, v. 19, n. 9, p. 1-15, [S.1.], 2017.

FILHO, A. B. C. et al. Objetos de Aprendizagem no Apoio à Aprendizagem de Engenharia: Explorando a Motivação Extrínseca. Novas Tecnologias na Educação, v. 10, n. 3, p. 1-10, 2012.

GOMES, A.; HENRIQUES, J.; MENDES, A. J. Uma proposta para ajudar alunos com dificuldades na aprendizagem inicial de Programação de Computadores. Educação, Formação \& Tecnologias, vol. 1, Centro de Informática e Sistemas da Universidade de Coimbra, [S.1.], 2008. Disponível em: $<$ http://www.eft.educom.pt/index.php/eft/artic le/view/23>. Acesso em: 27 out. 2017.

GUIMARÃES, C.; DUARTE, R. O. O ensino de Pesquisa Operacional em cursos de Sistema de Informação: uma abordagem prática Anais... Simpósio Brasileiro de Pesquisa Operacional, p. 410-421, 2007. Disponível em: < http://www.din.uem.br/sbpo/sbpo2007/pdf/arq 0004.pdf>. Acesso em: 27 out. 2017.
KITCHENHAM, B. Procedure for undertaking systematic reviews. Computer Science Department, Keele University and National ICT Australia, Joint Technical Report, 2004.

MOTA, R. P. B. Código livre Scilab para o ensino de Cálculo Numérico. Anais... XXII Simpósio Brasileiro de Informática na Educação Universidade Federal do Ceará (UFC), p. 600609, 2011. Disponível em: < http://www.brie.org/pub/index.php/sbie/article/viewFile/162 2/1387 >. Acesso em: 27 out. 2017.

MÜLLER, I. Tendências atuais de Educação Matemática. Revista de Ensino, Educação e Ciências Humanas, v. 1, n. 1, p. 133-144, 2000.

PAZETO, T. A.; PRIETCH, S. S. Experiências e Propostas de Projetos Interdisciplinares no Projeto Pedagógico de curso de Licenciatura em Computação. Anais... II Workshop de Licenciatura em Computação (Curso de Licenciatura Plena em Informática)- UFMT Campus de Rondonópolis, [S.1.], 2010. Disponível

em: <http://www.academia.edu/download/330 42246/WLC_2010_Interdisc_vsFinal.pdf $>$. Acesso em: 27 out. 2017.

PRETO, T. M. et al. Possibilidades para a integração entre Matemática e programação. Anais... XXXIII Congresso Brasileiro de Ensino em Engenharia. 2005. Disponível em: $<$ http://198.136.59.239/ abengeorg/CobengeA nteriores/2005/artigos/PR-5-102102698041118705867959.pdf>. Acesso em: 27 out. 2017.

PRETO, T. M.; SCHEER, S. Integração entre disciplinas da área de matemática e programação através de trabalhos interdisciplinares. Anais... Congresso Brasileiro de Ensino em Engenharia, 2004. Disponível em: <http://198.136.59.239/ abengeorg/Cobenge Anteriores/2004/artigos/01_592.pdf>. Acesso em: 27 out. 2017.

SILVA, S. R. X. O uso do Scilab como ferramenta para o ensino de cálculo numérico. Anais... XLI Congresso Brasileiro de Ensino de Engenharia. 2013. Disponível em: $<$ https://turing.pro.br/anais/COBENGE- 
2013/pdf/ 117997_1.pdf>. Acesso em: 27 out. 2017.

SILVA, A. J.; SOUZA, V. M.; SILVA, J. D. M. Linguagem de Programação Basic: aplicando no Ensino de Matemática. Colloquium Exactarum, v. 8, n. 3, p. 65-85, 2016.

SOUSA, E. D. Avaliação de um software para o ensino de pesquisa operacional: um estudo de caso. 2014. 132 f. Dissertação (Mestrado em Produção e Sistemas) - Pontifícia Universidade Católica de Goiás, Goiânia GO, 2014.
ZAINA, L. A. M.; CAVERSAN, F. L. Projeto multidisciplinar: uma experiência prática no ensino de programação em um curso de Engenharia da computação. Anais... XXXIII Congresso Brasileiro de Ensino de Engenharia. 2005. Disponível em: http://198.136.59.239/ abengeorg/CobengeA nteriores/2005/artigos/SP-15-285453218051118683317399.pdf>. Acesso em: 27 out. 2017.
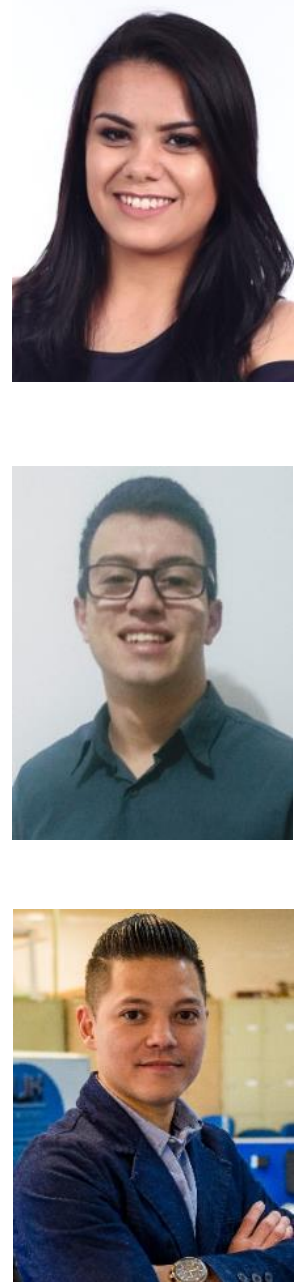

\section{DADOS BIOGRÁFICOS DOS AUTORES}

Karoline Guedes - Graduada em Engenharia de Produção pela Universidade Federal do Paraná (UFPR) em 2018. Mestranda em Engenharia de Produção pela Universidade Estadual de Maringá (UEM) desde 2020, bolsista da Coordenação de Aperfeiçoamento de Pessoal de Nível Superior (CAPES). Tem experiência nas áreas de Planejamento e Controle da Produção, Gestão de Operações Sustentáveis e Eficiência Energética.

Antônio Henrique Meira - Graduado em Engenharia de Produção pela Universidade Federal do Paraná (2019), participação de projetos de pesquisa de Iniciação Científica na área de Pesquisa Operacional aplicado a problemas logísticos nos anos de 2016-2019. Atualmente trabalha no Planejamento e Controle da Produção (PCP) de uma fábrica de espuma e colchões, exercendo as funções de controle de matéria prima, análise de tempo e movimentações e desenvolvendo sistemas de melhoria de produtividade.

Carlos Roberto Beleti Junior - Graduado em Ciência da Computação com ênfase em Engenharia de Software pela Universidade Estadual de Maringá (UEM) em 2009, Mestre em Ciência da Computação pelo Programa de Pós-graduação em Ciência da Computação (PCC-UEM) em 2013. Doutorando em Educação pelo Programa de Pós-graduação em Educação (PPE-UEM) desde 2020. Atualmente é Professor Assistente na Universidade Federal do Paraná, Campus Avançado em Jandaia do Sul. Atua nas áreas de ensino de Algoritmos e Programação, Arquitetura de Computadores e Divulgação Científica. 


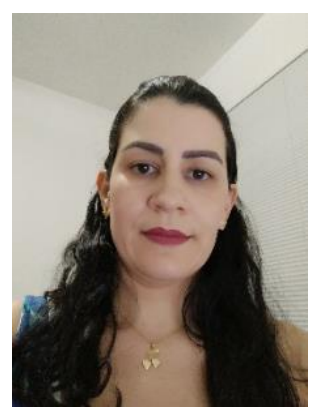

Juliana Verga Shirabayashi - Graduada em Matemática pela Universidade Estadual Paulista (2005), Mestre em Engenharia Elétrica pela Universidade Estadual de Campinas (2009) e Doutora em Engenharia Elétrica (2014) pela mesma universidade. Desde 2014 é Professora Adjunta na Universidade Federal do Paraná, Campus Avançado em Jandaia do Sul. É credenciada no Mestrado Profissional em Matemática Aplicada e Computacional - IMECC/UNICAMP e vice coordenadora da Regional 11 da Sociedade Brasileira de Matemática Aplicada e Computacional (SBMAC). É membro do comitê temático de Mulheres na Matemática Aplicada e Computacional (SBMAC). Atua em projetos de extensão em Educação Matemática desde 2014 e coordena projetos de pesquisa na área de Pesquisa Operacional e Internet das Coisas. Tem experiência na área de Pesquisa Operacional, atuando principalmente nos seguintes temas: otimização, problemas de rotas, problemas de corte, programação matemática fuzzy, grafos, problemas de transporte multimodal fuzzy, internet das coisas. 